\title{
Photoperiodic Flower Induction of Several Kalanchoe Species and Ornamental Characteristics of the Flowering Species
}

\author{
Christopher J. Currey and John E. Erwin ${ }^{1,2}$ \\ Department of Horticultural Science, University of Minnesota, 1970 Folwell \\ Avenue, St. Paul, MN 55108-6007
}

Additional index words. Kalanchoe laciniata, Kalanchoe manginii, Kalanchoe nyikae, Kalanchoe uniflora, Kalanchoe velutina, new ornamentals, total color index

\begin{abstract}
Our objectives in this study were to identify the flowering response of Kalanchoe spp. to photoperiodic treatments and characterize flowering and vegetative characteristics of flowering plants. Twenty vegetatively propagated Kalanchoe spp. were grown under one of four photoperiodic treatments: 1) short days (SD; 8-h photoperiod) for 16 weeks; 2) night interruption lighting (NI; 2000 to $0200 \mathrm{HR}$ ) for 16 weeks; 3) SD for 8 weeks then transferred to NI for 8 weeks; or 4) NI for 8 weeks then transferred to SD for 8 weeks. Kalanchoe beauvardii, K. behariensis, K. fedtschenkoi, K. longiflora, $K$. marmorata, $K$. marnieriana, $K$. streptantha, $K$. tomentosa, and $K$. vigueridoi did not flower under any treatment. Kalanchoe laetivirens and $K$. rosei had minimal flowering when exposed to NI followed by SD, whereas $K$. pumila had minimal flowering when exposed to SD followed by NI. Kalanchoe glaucescens, K. laciniata, K. manginii, K. nyikae, $K$. rotundifolia, $K$. uniflora, and $K$. velutina flowered when exposed to SD for 8 or 16 weeks, and node number below the inflorescence and days to first open flower for these species increased when NI preceded SD. Kalanchoe millotii flowered under a 16-week SD treatment only. No plants flowered when grown under only NI. We classified $K$. glaucescens, K. laciniata, K. manginii, $K$. millotii, $K$. nyikae, $K$. rotundifolia, $K$. uniflora, and $K$. velutina as obligate SD plants. Flower diameter, total flower number, total color index, shoot length, branch number, and leaf length and width varied among species. Based on these ornamental characteristics, we identified $K$. glaucescens, $K$. laciniata, $K$. manginii, $K$. nyikae, $K$. uniflora, and $K$. velutina as potential ornamental flowering potted plants.
\end{abstract}

The genus Kalanchoe includes 139 species indigenous to Madagascar, southern and eastern Africa, and, to a lesser extent, tropical Africa, the Arabian Peninsula, and southeastern Asia (Descoings, 2005; Gherig et al., 2001). One species, K. blossfeldiana von Poellnitz, is grown commercially as a flowering potted plant, whereas other species are grown as minor flowering or potted plants (Dole and Wilkins, 2005). Kalanchoe spp. are generally amenable to cultivation and propagate readily from stem and leaf cuttings or plantlets produced along leaf margins (Descoings, 2005). Additionally, Kalanchoe spp. can vary in flower color, size, number, and inflorescence structure as well as foliage size, shape, and color (C. Currey, personal observation).

Photoperiodic flower induction is a common mechanism for floral induction. Plants

\footnotetext{
Received for publication 21 July 2010. Accepted for publication 12 Oct. 2010

Mention of trade names in this publication does not imply endorsement by the Minnesota Agriculture Experiment Station of products named nor criticisms of similar products not named.

${ }^{1}$ Professor.

${ }^{2}$ To whom reprint requests should be addressed; e-mailerwin001@umn.edu.
}

that have potential as new ornamental potted or garden plants.

\section{Materials and Methods}

Propagation and propagule culture. Stock plants of 20 Kalanchoe spp. (Table 1) were started from rooted and unrooted cuttings obtained from two firms. Vegetative stock plants were maintained in a greenhouse with $22 \pm 1{ }^{\circ} \mathrm{C}$ and $18 \pm 1{ }^{\circ} \mathrm{C}$ day and night air temperature set points, respectively, under ambient irradiance conditions (St. Paul, MN, $45^{\circ} \mathrm{N}$ ) supplemented with $75 \mu \mathrm{mol} \cdot \mathrm{m}^{-2} \cdot \mathrm{s}^{-1}$ photosynthetic photon flux from high-pressure sodium lamps when ambient irradiance was less than $200 \mu \mathrm{mol} \cdot \mathrm{m}^{-2} \cdot \mathrm{s}^{-1}$ from 0700 to $0100 \mathrm{HR}$ (18-h photoperiod). Plants were irrigated as necessary with tap water and fertilized once weekly with tap water supplemented with water-soluble fertilizer (Excel 15N-2.2P12.5K Cal-Mag; The Scotts Co., Marysville, $\mathrm{OH})$ to provide the following at each irrigation (in $\mathrm{mg} \cdot \mathrm{L}^{-1}$ ): 200 nitrogen, 29 phosphorous, 167 potassium, 67 calcium, 27 magnesium, 1.0 iron, 0.5 manganese and zinc, 0.3 copper and boron, and 0.1 molybdenum. The $\mathrm{pH}$ and alkalinity of the tap water was 7.7 and $55 \mathrm{mg} \cdot \mathrm{L}^{-1}$, respectively.

On 8 Oct. 2008, cuttings of each species were harvested and the lowest set of leaves was removed from the cutting and cuttings were dipped in talc powder containing 1000 ppm indole-3-butyric acid (Hormodin 1; OHP Inc., Mainland, PA) to promote rooting. Cuttings were then placed in soilless high-porosity growing medium (SB500; SunGro Horticulture, Bellevue, WA) in 128-, 72-, or 50-cell plug trays with individual cell volumes of 25,59 , or $111 \mathrm{~mL}$, respectively (Table 1), to accommodate species of different sizes. Air temperature and irradiance were as described previously with a media temperature set point of $21{ }^{\circ} \mathrm{C}$ maintained with bottom-heat mats placed beneath the trays. Cuttings were handmisted daily and irrigated with water only as needed. After 1 week, cuttings were irrigated with tap water as needed and fertilized once weekly as previously described.

After rooting (4 weeks), cuttings were transplanted into $10.2-\mathrm{cm}$ plastic pots $(538-\mathrm{mL}$ volume) filled with a high-porosity growing medium (SB500; SunGro Horticulture). Pots were then placed in the same environment in which they were rooted without bottom heat or daily misting. Plants were irrigated with tap water as needed and fertilized once weekly as previously described.

Photoperiodic treatments. Four photoperiodic treatments were selected to elicit flowering responses previously reported for Kalanchoe spp. (Schwabe, 1985; Zeevaart, 1985; Zimmer, 1985). Two weeks after transplant, plants were pinched to two nodes and placed under one of the following photoperiod treatments: 1) SD for 16 weeks (achieved by pulling an opaque cloth over the plants at $1600 \mathrm{HR}$ and retracting it at $0800 \mathrm{HR}$ ); 2) NI for 16 weeks (natural daylength with $2 \mu \mathrm{mol} \cdot \mathrm{m}^{-2} \cdot \mathrm{s}^{-1}$ incandescent lamps from 2000 to $0200 \mathrm{HR}$; natural daylength varied from $9 \mathrm{~h} 24 \mathrm{~min}$ to $11 \mathrm{~h} 17 \mathrm{~min}$ ); 3) SD 
for 8 weeks then transferred to NI for 8 weeks (SD-NI); or 4) NI for 8 weeks then transferred to SD for 8 weeks (NI-SD).

The plants were grown in a glass-glazed greenhouse with an exhaust fan and evaporative-pad cooling and radiant hot-water heating controlled by an environmental computer (Maximizer Precision 10; Priva Computers Inc., Vineland Station, Ontario, Canada). The greenhouse day and night air temperature set points were $22 \pm 1{ }^{\circ} \mathrm{C}$ and $18 \pm 1{ }^{\circ} \mathrm{C}$, respectively. Irradiance and air temperature at canopy height were measured with a quantum sensor (Model QSO-SUN; Apogee Instruments Inc., Logan, UT) and thermocouples (Type E Wire chromega/constantan; Omega Engineering Inc., Stamford, CT), respectively, and connected to a data logger (CR10X; Campbell Scientific Inc., Logan, UT), which recorded averages every $10 \mathrm{~min}$. Environmental data are reported in Table 2. Plants were irrigated with tap water as needed and fertilized once weekly as previously described.

Data collection. Data were collected on the date of first flower opening, node number below the terminal inflorescence, terminal inflorescence flower number, axillary inflorescence number, axillary inflorescence flower number, flower diameter of the first open flower, leaf length and width of a fully expanded leaf, and shoot height from the base

Table 1. Species used in this study with indigenous distribution, source of original material, and tray size used for propagation identified.

\begin{tabular}{|c|c|c|c|}
\hline Species & Indigenous distribution ${ }^{2}$ & Source & $\begin{array}{l}\text { Tray size } \\
\text { (cell no.) }\end{array}$ \\
\hline K. beauvardii Hamet & Madagascar & $\mathrm{GW}^{\mathrm{y}}$ & 128 \\
\hline K. behariensis Drake & Madagascar & $\mathrm{AP}$ & 72 \\
\hline K. fedtschenkoi Hamet \& H. Perrier & Madagascar & AP & 128 \\
\hline $\begin{array}{l}\text { K. glaucescens Britten 'Freeling's } \\
\text { Sensation' }\end{array}$ & $\begin{array}{l}\text { Central and east Africa, Sudan, Ethiopia, } \\
\text { Somalia, Arabian Peninsula }\end{array}$ & GW & 128 \\
\hline K. laciniata (Linné) De Chandolle & $\begin{array}{l}\text { Morocco; east, south, and southwest } \\
\text { Africa; Arabian Peninsula }\end{array}$ & GW & 72 \\
\hline K. laetivirens Descoings & Madagascar & GW & 50 \\
\hline K. longiflora Schlechter ex J. M. Wood & Madagascar, Toliara & $\mathrm{AP}$ & 128 \\
\hline K. manginii Hamet \& H. Perrier & Madagascar & $\mathrm{AP}$ & 128 \\
\hline K. marmorata Baker & Central and east Africa & AP & 128 \\
\hline K. marnieriana $\mathrm{H}$. Jacobsen & Madagascar & AP & 128 \\
\hline K. millotii Hamet \& H. Perrier & Madagascar & GW & 72 \\
\hline K. nvikae Engler & Kenya, Tanzania & AP & 50 \\
\hline K. pumila Baker & Madagascar & AP & 128 \\
\hline K. rosei Baker & Madagascar & GW & 128 \\
\hline K. rotundifolia (Haworth) Haworth & $\begin{array}{l}\text { Central and east to south and } \\
\text { southwest Africa; Socotra }\end{array}$ & GW & 128 \\
\hline K. streptantha Baker & Madagascar & GW & 72 \\
\hline K. tomentosa Baker & Madagascar & $\mathrm{AP}$ & 128 \\
\hline K. uniflora (Stapf) Hamet & Madagascar & $\mathrm{AP}$ & 128 \\
\hline K. velutina Welwitsch ex Britten & Angola, Zimbabwe & GW & 50 \\
\hline K. viguieri Hamet \& H. Perrier & Madagascar & AP & 128 \\
\hline
\end{tabular}

${ }^{\mathrm{z} F r o m ~ D e s c o i n g s, ~} 2005$.

yPlant sources were Altman Plants (AP; Vista, CA) or Glasshouse Works (GW; Stewart, OH).

Table 2. Mean day and night air temperature and daily light integral (DLI) for short-day (SD) night interruption (NI), SD followed by NI (SD-NI), and NI followed by SD (NI-SD) photoperiodic treatments.

\begin{tabular}{lccc}
\hline & \multicolumn{2}{c}{ Air temp $\left[\right.$ mean \pm SD $\left.\left({ }^{\circ} \mathrm{C}\right)\right]$} & \\
\cline { 2 - 3 } Treatment & Day & Night & DLI $\left(\mathrm{mol} \cdot \mathrm{m}^{-2} \cdot \mathrm{d}^{-1}\right)$ \\
\hline SD & $23.1 \pm 2.9$ & $19.4 \pm 2.3$ & 4.3 \\
NI & $22.6 \pm 2.8$ & $18.9 \pm 1.4$ & 4.8 \\
SD-NI & $22.8 \pm 2.7$ & $18.1 \pm 1.6$ & 4.5 \\
NI-SD & $22.7 \pm 2.9$ & $18.3 \pm 1.8$ & 4.4 \\
\hline
\end{tabular}

ence $(P \leq 0.05)$ were performed on all data using SPSS 16.0 (SPSS Inc., Chicago, IL). Analysis of percentage data were performed on the arcsine of the square root of percentages (Little and Hills, 1978). Only plants in a treatment with a percentage population flowering significantly greater than zero were analyzed for node number below the inflorescence and days to flower. Only plants flowering under SD were analyzed for ornamental characteristics. There were no differences in environmental, flower induction, and ornamental data between replications so data were pooled.

\section{Results}

Flower induction and development. Species and photoperiod interacted to affect percent flowering plants. $K$. beauvardii, $K$. behariensis, $K$. fedtschenkoi, $K$. longiflora, $K$. marmorata, $K$. marnieriana, $K$. streptantha, $K$. tomentosa, and $K$. vigueridoi did not flower under any treatment (Table 3). Although not significantly different from nonflowering $(0 \%)$ populations, $9 \%$ of $K$. pumila flowered under SD followed by NI, whereas $2 \%$ and $25 \%$ of $K$. laetivirens and $K$. rosei, respectively, flowered under NI followed by $\mathrm{SD}$. No flowering occurred when $K$. glaucescens, K. laciniata, K. manginii, K. millotii, $K$. nyikae, $K$. rotundifolia, $K$. uniflora, and $K$. velutina were grown under continuous NI. One hundred percent of $K$. glaucescens, $K$. laciniata, K. manginii, $K$. nyikae, $K$. rotundifolia, $K$. uniflora, and $K$. velutina flowered under continuous SD, SD followed by NI, and NI followed by SD, whereas $61 \%$ of K. millotii flowered under SD (Table 3).

Node number was affected by species and photoperiod differently. For instance, under SD followed by NI, K. manginii and $K$. velutina had seven nodes below the terminal inflorescence, whereas under NI followed by SD node number below the inflorescence was 12 and eight nodes, respectively (Table 4). There were four nodes below the terminal inflorescence of $K$. millotii under SD (Table $4)$. Node number below the terminal inflorescence of $K$. glaucescens, $K$. laciniata, $K$. manginii, $K$. nyikae, $K$. rotundifolia, $K$. uniflora, and $K$. velutina increased when grown under NI preceded by SD compared with plants grown under SD or SD followed by NI and (Table 4).

Species and photoperiod interacted to affect days to first open flower. The days to first open flower of $K$. glaucescens, $K$. laciniata, $K$. manginii, $K$. nyikae, $K$. rotundifolia, $K$. uniflora, and $K$. velutina under SD or SD followed by NI were fewer when compared with plants grown under NI followed by SD (Table 5). For example, days to first open flower for $K$. laciniata and $K$. velutina grown under SD were 90 and $91 \mathrm{~d}$, respectively, and increased to 143 and $133 \mathrm{~d}$, respectively, when grown under NI followed by SD (Table 4); K. millotii flowered in $107 \mathrm{~d}$ when grown under SD (Table 5).

Ornamental characteristics. Among species that flowered, species varied in flower 
Table 3. The percent population flowering of 20 Kalanchoe spp. grown under short-day (SD) for 16 weeks (achieved by pulling an opaque cloth over the plants from 1600 to $0800 \mathrm{HR}$; 8-h photoperiod), night interruption (NI; $2 \mu \mathrm{mol} \cdot \mathrm{m}^{-2} \cdot \mathrm{s}^{-1}$ incandescent light from 2000 to $0200 \mathrm{HR}$ ) for 16 weeks, SD for 8 weeks then transferred to NI for 8 weeks (SD-NI), or NI for 8 weeks then transferred to SD for 8 wks $(\mathrm{NI}-\mathrm{SD})^{\mathrm{z}}$.

\begin{tabular}{lrrrr}
\hline & \multicolumn{4}{c}{ Percentage population flowering (\%) } \\
\cline { 2 - 5 } Species & \multicolumn{1}{c}{$\mathrm{SD}$} & $\mathrm{NI}$ & $\mathrm{SD}-\mathrm{NI}$ & $\mathrm{NI}-\mathrm{SD}$ \\
\hline K. beauvardii & $0^{\mathrm{y}} \mathrm{a}^{\mathrm{x}} \mathrm{A}^{\mathrm{w}}$ & $0 \mathrm{aA}$ & $0 \mathrm{aA}$ & $0 \mathrm{aA}$ \\
K. behariensis & $0 \mathrm{aA}$ & $0 \mathrm{aA}$ & $0 \mathrm{aA}$ & $0 \mathrm{aA}$ \\
K. fedtschenkoi & $0 \mathrm{aA}$ & $0 \mathrm{aA}$ & $0 \mathrm{aA}$ & $0 \mathrm{aA}$ \\
K. glaucescens & $100 \mathrm{bC}$ & $0 \mathrm{aA}$ & $100 \mathrm{bD}$ & $100 \mathrm{bD}$ \\
K. laciniata & $100 \mathrm{bC}$ & $0 \mathrm{aA}$ & $100 \mathrm{aD}$ & $100 \mathrm{bD}$ \\
K. laetivirens & $0 \mathrm{aA}$ & $0 \mathrm{aA}$ & $0 \mathrm{aA}$ & $2 \mathrm{aAB}$ \\
K. longiflora & $0 \mathrm{aA}$ & $0 \mathrm{aA}$ & $0 \mathrm{aA}$ & $0 \mathrm{aA}$ \\
K. manginii & $100 \mathrm{bC}$ & $0 \mathrm{aA}$ & $100 \mathrm{bD}$ & $100 \mathrm{bD}$ \\
K. marmorata & $0 \mathrm{aA}$ & $0 \mathrm{aA}$ & $0 \mathrm{aA}$ & $0 \mathrm{aA}$ \\
K. marnieriana & $0 \mathrm{aA}$ & $0 \mathrm{aA}$ & $0 \mathrm{aA}$ & $0 \mathrm{aA}$ \\
K. millotii & $61 \mathrm{bB}$ & $0 \mathrm{aA}$ & $47 \mathrm{abC}$ & $47 \mathrm{abC}$ \\
K. nyikae & $100 \mathrm{bC}$ & $0 \mathrm{aA}$ & $100 \mathrm{bD}$ & $100 \mathrm{bD}$ \\
K. pumila & $0 \mathrm{aA}$ & $0 \mathrm{aA}$ & $9 \mathrm{aB}$ & $0 \mathrm{aA}$ \\
K. rosei & $0 \mathrm{aA}$ & $0 \mathrm{aA}$ & $0 \mathrm{aA}$ & $25 \mathrm{aBC}$ \\
K. rotundifolia & $100 \mathrm{bC}$ & $0 \mathrm{aA}$ & $100 \mathrm{bD}$ & $100 \mathrm{bD}$ \\
K. streptantha & $0 \mathrm{aA}$ & $0 \mathrm{aA}$ & $0 \mathrm{aA}$ & $0 \mathrm{aA}$ \\
K. tomentosa & $0 \mathrm{aA}$ & $0 \mathrm{aA}$ & $0 \mathrm{aA}$ & $0 \mathrm{aA}$ \\
K. uniflora & $100 \mathrm{bC}$ & $0 \mathrm{aA}$ & $100 \mathrm{bD}$ & $100 \mathrm{bD}$ \\
K. velutina & $100 \mathrm{bC}$ & $0 \mathrm{aA}$ & $100 \mathrm{bD}$ & $100 \mathrm{bD}$ \\
K. vigueridoi & $0 \mathrm{aA}$ & $0 \mathrm{aA}$ & $0 \mathrm{aA}$ & $0 \mathrm{aA}$ \\
Species & $* * * \mathrm{v}$ & & & \\
Photoperiod & $* * *$ & & & \\
Species $\times$ photoperiod & $* * *$ & & & \\
\hline
\end{tabular}

${ }^{\mathrm{z}}$ Analysis of percentage data were performed on the arcsine of the square root of percentages (backtransformed data are presented).

${ }^{y}$ Numerals represent treatment mean.

${ }^{x}$ Lower case letters indicate mean separations across photoperiods, within a species, by Tukey's honestly significant difference (HSD) test at $P \leq 0.05$.

"Upper case letters indicate mean separations across species, within a photoperiod, by Tukey's HSD test at $P \leq 0.05$.

v***Significant at $P \leq 0.001$.

Table 4. Node number below the terminal inflorescence of $K$. glaucescens, $K$. laciniata, $K$. laetivirens, $K$. manginii, K. millotii, K. nyikae, $K$. pumila, $K$. rosei, K. rotundifolia, $K$. uniflora, and $K$. velutina grown under short-day (SD) for 16 weeks (achieved by pulling an opaque cloth over the plants from 1600 to $0800 \mathrm{HR} ; 8$-h photoperiod), night interruption (NI; $2 \mu \mathrm{mol} \cdot \mathrm{m}^{-2} \cdot \mathrm{s}^{-1}$ incandescent light from 2000 to 0200 HR) for 16 weeks, SD for 8 weeks then transferred to NI for 8 weeks (SD-NI), or NI for 8 weeks then transferred to SD for 8 weeks (NI-SD). ${ }^{\mathrm{z}}$

\begin{tabular}{llrcc}
\hline & \multicolumn{4}{c}{ Nodes below the terminal inflorescence (no.) } \\
\cline { 2 - 5 } Species & $\mathrm{SD}$ & $\mathrm{NI}$ & $\mathrm{SD}-\mathrm{NI}$ & $\mathrm{NI}-\mathrm{SD}$ \\
\hline K. glaucescens & $6^{\mathrm{y}} \mathrm{a}^{\mathrm{x} B \mathrm{BC}^{\mathrm{w}}}$ & $(17)^{\mathrm{v}}$ & $6 \mathrm{aA}$ & $14 \mathrm{bDE}$ \\
K. laciniata & $8 \mathrm{aD}$ & $(8)$ & $8 \mathrm{aB}$ & $13 \mathrm{bCD}$ \\
K. manginii & $6 \mathrm{aB}$ & $(13)$ & $7 \mathrm{aA}$ & $12 \mathrm{bC}$ \\
K. millotii & $4 \mathrm{~A}$ & $(6)$ & $(6)$ & $(6)$ \\
K. nyikae & $7 \mathrm{aC}$ & $(6)$ & $8 \mathrm{aB}$ & $10 \mathrm{bB}$ \\
K. rotundifolia & $6 \mathrm{aB}$ & $(17)$ & $6 \mathrm{aA}$ & $16 \mathrm{bF}$ \\
K. uniflora & $6 \mathrm{aB}$ & $(15)$ & $6 \mathrm{aA}$ & $12 \mathrm{bC}$ \\
K. velutina & $6 \mathrm{aB}$ & $(4)$ & $7 \mathrm{aA}$ & $8 \mathrm{bA}$ \\
Species & $* * * u$ & & & \\
Photoperiod & $* * *$ & & & \\
Species $\times$ photoperiod & $* * *$ & & & \\
\hline Ony & & &
\end{tabular}

${ }^{2}$ Only flowering plants were analyzed.

${ }^{y}$ Numerals represent treatment mean.

${ }^{\mathrm{x}}$ Lower case letters indicate mean separations across photoperiods, within a species, by Tukey's honestly significant difference (HSD) test at $P \leq 0.05$.

"Upper case letters indicate mean separations across species, within a photoperiod, by Tukey's HSD test at $P \leq 0.05$.

"Numerals in parentheses represent node number at the end of experiment on plants that did not flower. u***Significant at $P \leq 0.001$.

diameter, total flower number, and total color grown under SD. Flower diameter ranged from $8 \mathrm{~mm}$ to $28 \mathrm{~mm}$ for $K$. millotii and K. laciniata, respectively (Table 6). Flower number ranged from 29 flowers to 143 flowers for $K$. uniflora and $K$. laetivirens, respectively (Table 6). The total color index of flowering
The number of branches varied from 0.0 branches for $K$. glaucescens, $K$. millotii, $K$. nyikae, and $K$. rotundifolia to 0.8 branches for $K$. laciniata (Table 7). Leaf length and width ranged from $4.8 \mathrm{~cm}$ and $1.9 \mathrm{~cm}$, respectively, for $K$. uniflora to $14.7 \mathrm{~cm}$ and $13.1 \mathrm{~cm}$, respectively, for K. laciniata (Table 7).

\section{Discussion}

Identifying flower induction requirements of Kalanchoe spp. with potential as new ornamental crops is a necessary step in commercialization (Wilkins and Erwin, 1998). In this study, $100 \%$ flowering was achieved for eight species, minimal flowering in two species, and no flowering in nine species. Kalanchoe glaucescens, $K$. laciniata, $K$. manginii, $K$. nyikae, $K$. rotundifolia, $K$. uniflora, and $K$. velutina all flowered when exposed to $\mathrm{SD}$ for 8 weeks or longer but did not flower when grown under NI only (Table 3 ). There was no difference in the number of nodes below the terminal inflorescence (Table 4) or days to first open flower (Table 5) for $K$. glaucescens, $K$. laciniata, $K$. manginii, $K$. nyikae, $K$ rotundifolia, $K$. uniflora, and $K$. velutina grown under SD versus SD followed by NI, suggesting plants were induced at the same point in time. However, when NI preceded SD, there was an increase in node number below the terminal inflorescence (Table 4) and days to first open flower (Table 5) on K. glaucescens, K. laciniata, K. manginii, K. nyikae, K rotundifolia, K. uniflora, and $K$. velutina suggesting NI delayed flower induction. K. millotii flowered under SD but not NI. Taken together, we conclude that these species can be classified as obligate SDP based on the duration of exposure to different photoperiodic treatments used in this experiment.

The classification of these Kalanchoe spp. as SDP agrees with work on other Kalanchoe spp., which were classified as SDP (Schwabe, 1985; Zimmer, 1985). However, our classification of $K$. velutina as flowering in response to $\mathrm{SD}\left(19.4{ }^{\circ} \mathrm{C}\right.$ average night temperature $)$ contradicts results of Sharma (1973) in which flowering of $K$. velutina was induced by a $15.5^{\circ} \mathrm{C}$ night temperature, demonstrating plants could be vernalized. It is not uncommon for species to have multiple flowering pathways, as observed in Arabidopsis thaliana (L.) Heynh. (Simpson and Dean, 2002).

Heat delay is a common physiological disorder associated with inhibition of photoperiodic flower induction of $K$. blossfeldiana, occurring when night temperatures during SD skotoperiods exceed $27{ }^{\circ} \mathrm{C}$ (Pertuit, 1977). The low percentages of $K$. laetivirens and $K$. rosei flowering under the NI followed by SD treatment may be the result of supraoptimal temperatures during NI $\left(18.3^{\circ} \mathrm{C}\right)$. $K$. daigremontiana, a LSDP, flowered under $\mathrm{SD}$ at $23{ }^{\circ} \mathrm{C}$ when the preceding $\mathrm{LD}$ air temperature was 11 to $15^{\circ} \mathrm{C}$ but not $19{ }^{\circ} \mathrm{C}$ (Zeevaart, 1985). This may indicate a need for cooler temperatures during the LD phase to increase the percentages of $K$. laetivirens and $K$. rosei plants flowering. 
Table 5. Days to first open flower of flowering Kalanchoe glaucescens, $K$. laciniata, $K$. laetivirens, $K$. manginii, K. millotii, $K$. nyikae, $K$. pumila, $K$. rosei, $K$. rotundifolia, $K$. uniflora, and $K$. velutina grown under short-day (SD) for 16 weeks (achieved by pulling an opaque cloth over the plants from 1600 to $0800 \mathrm{HR} ; 8$-h photoperiod), night interruption (NI; $2 \mu \mathrm{mol} \cdot \mathrm{m}^{-2} \cdot \mathrm{s}^{-1}$ incandescent light from 2000 to 0200 HR) for 16 weeks, SD for 8 weeks then transferred to NI for 8 weeks (SD-NI), or NI for 8 weeks then transferred to SD for 8 weeks (NI-SD). ${ }^{\mathrm{z}}$

\begin{tabular}{lcccc}
\hline & \multicolumn{4}{c}{ Days to first open flower (no.) } \\
\cline { 2 - 5 } Species & \multicolumn{1}{c}{ SD } & NI & SD-NI & NI-SD \\
\hline K. glaucescens & $74^{\mathrm{y}} \mathrm{a}^{\mathrm{x}} \mathrm{B}^{\mathrm{w}}$ & - & $72 \mathrm{aA}$ & $116 \mathrm{bA}$ \\
K. laciniata & $94 \mathrm{aE}$ & - & $90 \mathrm{aBC}$ & $143 \mathrm{bC}$ \\
K. manginii & $87 \mathrm{aC}$ & - & $86 \mathrm{aB}$ & $132 \mathrm{bC}$ \\
K. millotii & $107 \mathrm{~F}$ & - & - & - \\
K. nyikae & $88 \mathrm{aCD}$ & - & $88 \mathrm{aBC}$ & $134 \mathrm{bB}$ \\
K. rotundifolia & $67 \mathrm{aA}$ & - & $67 \mathrm{aA}$ & $115 \mathrm{bA}$ \\
K. uniflora & $92 \mathrm{aDE}$ & - & $90 \mathrm{aBC}$ & $133 \mathrm{bB}$ \\
K. velutina & $91 \mathrm{aCDE}$ & - & $90 \mathrm{aC}$ & $133 \mathrm{bB}$ \\
Species & $* * * u$ & & \\
Photoperiod & $* * *$ & & & \\
Species $\times$ photoperiod & $* * *$ & & & \\
\hline On & & & &
\end{tabular}

${ }^{\mathrm{z}}$ Only flowering plants were analyzed.

${ }^{y}$ Numerals represent treatment mean.

${ }^{\mathrm{x}}$ Lower case letters indicate mean separations across photoperiods, within a species, by Tukey's honestly significant difference (HSD) test at $P \leq 0.05$.

wUpper case letters indicate mean separations across species, within a photoperiod, by Tukey's HSD test at $P \leq 0.05$.

vIndicates plants did not flower

u*** Significant at $P \leq 0.001$.

Table 6. Flowering characteristics including flower diameter, total flower number, total color index, flower color and notes of flowering Kalanchoe glaucescens, $K$. laciniata, $K$. laetivirens, $K$. manginii, $K$. nyikae, K. pumila, K. rosei, K. rotundifolia, K. uniflora, and K. velutina grown under short-day for 16 weeks (achieved by pulling an opaque cloth over the plants from 1600 to $0800 \mathrm{HR}$; 8-h photoperiod).

\begin{tabular}{|c|c|c|c|c|c|}
\hline Species & $\begin{array}{c}\text { Flower } \\
\text { diam }(\mathrm{mm})\end{array}$ & $\begin{array}{l}\text { Total flower } \\
\text { number (no.) }\end{array}$ & $\begin{array}{l}\text { Total color } \\
\text { index }\left(\mathrm{cm}^{2}\right)\end{array}$ & $\begin{array}{l}\text { Flower } \\
\text { color }\end{array}$ & Notes \\
\hline $\bar{K}$.glaucescens & $8^{z} a^{y}$ & $58 \mathrm{bc}$ & $31 \mathrm{a}$ & Orange & \\
\hline K. laciniata & $28 \mathrm{f}$ & $59 \mathrm{bc}$ & $364 \mathrm{c}$ & Yellow & \\
\hline K. manginii & $14 \mathrm{c}$ & $43 \mathrm{ab}$ & $69 \mathrm{a}$ & Red & Pendant flowers \\
\hline K. millotii & $8 \mathrm{a}$ & $104 \mathrm{e}$ & $58 \mathrm{a}$ & White & $\begin{array}{l}\text { Inflated calyces obscure } \\
\text { corollas }\end{array}$ \\
\hline K. nyikae & $23 \mathrm{e}$ & 79 cde & $353 \mathrm{c}$ & Pink & \\
\hline K. rotundifolia & $11 \mathrm{~b}$ & $75 \mathrm{~cd}$ & $75 \mathrm{a}$ & Orange & Pendant flowers \\
\hline K. uniflora & $16 \mathrm{~d}$ & $29 \mathrm{a}$ & $58 \mathrm{a}$ & Salmon & \\
\hline K. velutina & $16 \mathrm{~d}$ & $100 \mathrm{de}$ & $209 \mathrm{~b}$ & Orange & \\
\hline Species & $* * * \mathrm{x}$ & $* * *$ & $* * *$ & & \\
\hline
\end{tabular}

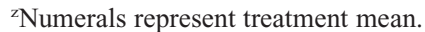

${ }^{y}$ Within-column means followed by different letters are significantly different by Tukey's honestly significant difference test at $P \leq 0.05$.

x*** Significant at $P \leq 0.001$.

Table 7. Vegetative characteristics including shoot height, branch number, leaf length, leaf width, leaf shape of flowering $K$. glaucescens, $K$. laciniata, K. laetivirens, $K$. manginii, $K$. nyikae, $K$. pumila, $K$. rosei, $K$. rotundifolia, $K$. uniflora, and $K$. velutina grown under short-day for 16 weeks (achieved by pulling an opaque cloth over the plants from 1600 to $0800 \mathrm{HR}$; 8-h photoperiod).

\begin{tabular}{lccccll}
\hline Species & $\begin{array}{c}\text { Shoot ht } \\
(\mathrm{cm})\end{array}$ & $\begin{array}{c}\text { Branch } \\
\text { number (no.) }\end{array}$ & $\begin{array}{c}\text { Leaf } \\
\text { length }(\mathrm{cm})\end{array}$ & $\begin{array}{c}\text { Leaf } \\
\text { width }(\mathrm{cm})\end{array}$ & Leaf shape ${ }^{\mathrm{z}}$ & \multicolumn{1}{c}{ Notes } \\
\hline K. glaucescens & $13.3^{\mathrm{y}} \mathrm{a}^{\mathrm{x}}$ & $0.0 \mathrm{a}$ & $5.2 \mathrm{a}$ & $2.5 \mathrm{ab}$ & Ovate & White margin on leaf \\
K. laciniata & $27.3 \mathrm{c}$ & $0.8 \mathrm{~b}$ & $14.7 \mathrm{c}$ & $13.1 \mathrm{e}$ & Dissected & \\
K. manginii & $20.6 \mathrm{~b}$ & $0.2 \mathrm{ab}$ & $5.5 \mathrm{a}$ & $3.3 \mathrm{abc}$ & Ovate & \\
K. millotii & $16.9 \mathrm{ab}$ & $0.0 \mathrm{a}$ & $7.1 \mathrm{a}$ & $4.3 \mathrm{bcd}$ & Ovate & Pubescent \\
K. nyikae & $33.9 \mathrm{~d}$ & $0.0 \mathrm{a}$ & $10.9 \mathrm{~b}$ & $6.1 \mathrm{~d}$ & Sub-orbicular & Purple-green leaves \\
K. rotundifolia & $21.5 \mathrm{~b}$ & $0.0 \mathrm{a}$ & $6.2 \mathrm{a}$ & $3.3 \mathrm{abc}$ & Oblong & \\
K. uniflora & $12.6 \mathrm{a}$ & $0.1 \mathrm{ab}$ & $4.8 \mathrm{a}$ & $1.9 \mathrm{a}$ & Elliptical & \\
K. velutina & $30.2 \mathrm{~d}$ & $0.3 \mathrm{~b}$ & $7.1 \mathrm{a}$ & $5.1 \mathrm{~cd}$ & Sub-orbicular & Pubescent \\
Species & $* * * \mathrm{w}$ & $* * *$ & $* * *$ & $* * *$ & & \\
\hline
\end{tabular}

${ }^{2}$ From Descoings, 2005.

${ }^{\mathrm{y}}$ Numerals represent treatment mean.

${ }^{x}$ Within-column means followed by different letters are significantly different by Tukey's honestly significant difference test at $P \leq 0.05$.

w*** Significant at $P \leq 0.001$.

The lack of complete flowering on $K$. beauvardii, K. behariensis, K. fedtschenkoi, K. longiflora, K. marmorata, K. marnieriana, $K$. streptantha, $K$. tomentosa, and $K$. vigueridoi grown from seed, Zeevaart (1985) reported $K$. pinnata and $K$. daigremontiana flowered after 37 leaf pairs and 10 to 15 leaf pairs were present, respectively, suggesting an extended developmental period before flower induction. Regardless of the reason for lack of flowering for these species, the fact that these species do not flower in a 16-week timeframe effectively excludes their commercial potential; commercial potted crops are required to have a relatively short production period under standard greenhouse conditions.

Flower colors observed in this study (Table 6) were consistent with the range in flower color observed in $K$. blossfeldiana cultivars (Descoings, 2005; Leonard and Nell, 2000). Total flower number of any species in this study did not exceed those previously reported for K. blossfeldiana (Schwabe, 1985). $K$. manginii and $K$. uniflora flowers had an attractive pendant habit that differs from the inflorescence of $K$. blossfeldiana, whereas $K$. millotii were less attractive because the white corollas were obscured by inflated green calyces. $K$. laciniata and $K$. nyikae produced the most striking display of flowers, mainly as a result of flower size and total color (C. Currey, personal observation).

Shoot length varied widely among the flowering species (Table 7), from short species such as $K$. glaucescens and $K$. uniflora to the taller ones including $K$. nyikae and $K$. velutina. Therefore, greater height control will likely be required for species with increased finishing heights. Additionally, if grown under non-inductive NI conditions, internode number below the terminal inflorescence will increase, although internode length of $\mathrm{K}$. laciniata, $K$. manginii, $K$. millotii, $K$. nyikae, $K$. uniflora, and $K$. velutina was shorter under NI than SD; photoperiod did not affect internode length of $K$. glaucescens and $K$. rotundifolia (data not shown).

In general, the number of branches on flowering plants was low and did not add to the "fullness" of the crop. As a result, increasing branching chemically with plant growth regulators such as ethephon [(2-chloroethyl) phosphonic acid] or benzyladenine $[N-$ (phenylmethyl)-1H-purin-6-amine] or mechanically with pinching may be necessary to produce plants with branching similar to the free-branching modern $K$. blossfeldiana cultivars. Variation in leaf dimensions, shape, color, and/or texture has the potential to increase the variety of vegetative characteristics of commercially produced flowering $\mathrm{Ka}$ lanchoe. In addition, foliage of some species may warrant their use as ornamental foliage plants. The shape and size of $K$. laciniata and $K$. nyikae foliage, coloration of $K$. glaucescens and $K$. nyikae foliage, and leaf surface of $K$. velutina foliage are all unique and attractive (C. Currey, personal observation).

\section{Conclusions}

Based on the flower induction and development data, we classify $K$. glaucescens, K. laciniata, K. manginii, K. millotii, K. nyikae, $K$. rotundifolia, $K$. uniflora, and $K$. velutina as 
obligate SD plants. We were unable to classify $K$. laetivirens, $K$. pumila, and $K$. rosei as a result of minimal flowering. The requirements for flowering of $K$. beauvardii, $K$. behariensis, $K$ fedtschenkoi, K. longiflora, K. marmorata, $K$. marnieriana, $K$. streptantha, $K$. tomentosa, and $K$. vigueridoi are not understood. We identified six species $K$. glaucescens 'Freeling's Sensation', K. laciniata, K. manginii, $K$. nyikae, $K$. uniflora, and $K$. velutina with flowering and/or vegetative characteristics that have ornamental potential as flowering potted plants.

\section{Literature Cited}

Descoings, B. 2005. Kalanchoe, p. 143-181. In: Eggli, E. (ed.). Illustrated handbook of succulent plants: Crassulaceae, Springer, Berlin, Germany.

Dole, J.M. and H.F. Wilkins. 2005. Kalanchoe, p. 629-635. In: Dole, J.M. and H.F. Wilkins (eds.). Floriculture: Principles and species. 2nd
Ed. Pearson Prentice Hall, Upper Saddle River, NJ.

Gherig, H., O. Gaußmann, H. Marx, D. Schwarzott, and M. Kluge. 2001. Molecular phylogeny of the genus Kalanchoe (Crassulaceae) inferred from nucleotide sequences of the ITS- 1 and ITS-2 regions. Plant Sci. 160:827-835.

Leonard, R.T. and T.A. Nell. 2000. Effects of production and postproduction factors on longevity and quality of Kalanchoe. Acta Hort. 518 : 121-124.

Little, T.M. and F.J. Hills. 1978. Transformations, p. 139-166. Agricultural experimentation: Design and analysis. John Wiley and Sons, New York, NY.

Pertuit, A.J., Jr. 1977. Influence of temperatures during long-night exposures on growth and flowering of 'Mace', 'Thor', and 'Telstar' Kalanchoe. HortScience 12:48-49.

Roh, M.S. and R.H. Lawson. 1998. Requirements for new floral crops-Perspectives for the United States of America. Acta Hort. 454: 29-38.
Schwabe, W.W. 1985. Kalanchoe blossfeldiana, p. 217-235. In: Halevy, A.H. (ed.). Handbook of flowering. Vol. III. CRC Press, Boca Raton, FL.

Sharma, G.K. 1973. Flower formation in Kalanchoe velutina induced by low night temperature. Southwest. Nat. 18:331-334.

Simpson, G.G. and C. Dean. 2002. Arabidopsis, the rosetta stone of flowering time? Science 296: 285-289.

Thomas, B. and D. Vince-Prue. 1997. Photoperiodic control of flower initiation: Some general principles, p. 3-28. In: Photoperiodism in plants. 2nd Ed. Academic Press, San Diego, CA.

Wilkins, H.F. and J.E. Erwin. 1998. Necessary considerations to introduce a new taxa. Acta Hort. 454:81-83.

Zeevaart, J.A. 1985. Bryophyllum, p. 89-100. In: Halevy, A.H. (ed.). Handbook of flowering. Vol. II. CRC Press, Boca Raton, FL.

Zimmer, K. 1985. Kalanchoe porphyrocalyx, p. 236239. In: Halevy, A.H. (ed.). Handbook of flowering. Vol. III. CRC Press, Boca Raton, FL. 